\title{
CARACTERIZACIÓN DE LAS PRINCIPALES PROBLEMÁTICAS DE LAS JUNTAS DE ACCIÓN COMUNAL (JAC) DEL MUNICIPIO DE GARAGOA
}

\section{CHARACTERIZATION OF THE MAIN PROBLEMS OF}

THE COMMUNITY ACTION MEETINGS (JAC) OF THE MUNICIPALITY OF GARAGOA

\author{
Autor 1 \\ Johana Rodríguez Huertas \\ Administradora de Empresas, Especialista en Gestión Pública, UNAD, Líder \\ semillero de investigación Líderes en Acción Correo electrónico: \\ ORCID: 0000-0002-2064-8520 \\ Correo electrónico: johana.rodriguez@unad.edu.co
}

\section{Autor 2}

Luzmina Lucia Buitrago Contreras

Estudiante Administración de Empresas, UNAD, Semillero de investigación Correo electrónico: luzmibuco0211@gmail.com

\section{RESUMEN}

Las Juntas de Acción Comunal, se constituyen como un medio de activo y organizado de participación ciudadana, con el propósito de realizar actividades de planeación y ejecución de políticas y programas para promover el desarrollo de las comunidades, en el escenario local.

En la actualidad estas organizaciones enfrentan diversas problemáticas que han debilitado su gestión administrativa, financiera, social, de participación y credibilidad frente a sus comunidades, las JAC en el municipio de Garagoa, no son ajenas a esto, por lo cual se requiere una caracterización de estas y sus principales problemas y 
debilidades, para desde de los resultados proponer estrategias que permitan su fortalecimiento en aspectos como competitividad y sostenibilidad, para el estudio planteamos la siguiente pregunta de investigación: ¿Caracterizar las juntas de acción comunal del municipio de Garagoa y sus principales problemáticas, permitirá generar estrategias para mejorar la gestión administrativa, organizativa y financiera de las mismas?

El objetivo es caracterizar las Juntas de Acción Comunal del municipio de Garagoa con el propósito de identificar las principales problemáticas, diagnosticarlas, analizarlas y generar estrategias que permitan aumentar su capacidad de gestión administrativa y financiera, liderazgo, participación, y credibilidad frente a sus comunidades.

Palabras clave: Asociación; Comunidad; participación; liderazgo; participación ciudadana; caracterización

\section{ABSTRACT}

The Community Action Boards have become a means of active, organized and conscious participation about the planning, evaluation and execution of development policies and programs for the communities, at the local stage.

Now a days, these organizations face several problems that have weakened their administrative, financial, social, participation and credibility management towards their communities, the JACs in the municipality of Garagoa, has not been totally inmune to this, so a characterization of these and its main problems and weaknesses, from the results propose strategies that allow its strengthening in aspects such as competitiveness and sustainability, for the study we pose the following research question: ¿Characterize the community action boards of the municipality of Garagoa and its main problems, will let generate strategies to improve the administrative, organizational and financial management of them?

The objective is to characterize the Community Action Boards of the municipality of Garagoa with the purpose of identifying the main problems, diagnosing them, analyzing them and generating strategies that allow increasing their administrative 


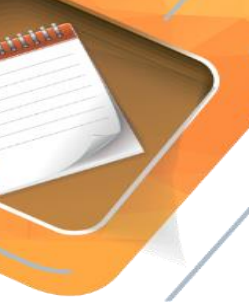

and financial management capacity, leadership, participation, and credibility towards their communities.

Keywords: Association; Community; participation; leadership; citizen participation; characterization.

\section{INTRODUCCIÓN}

En Colombia la acción comunal es una expresión ordenada de la sociedad civil que busca promover el desarrollo de las comunidades en su territorio. Según el Ministerio del Interior, es la forma de organización ciudadana para el desarrollo social y comunitario de mayor tradición en Colombia. (DNP, 2018, pág. 3)

Según la Ley 743 de 2002, en el artículo sexto, las organizaciones comunales se definen como: "una expresión social organizada, autónoma y solidaria de la sociedad civil, cuyo propósito es promover un desarrollo integral, sostenible y sustentable construido a partir del ejercicio de la democracia participativa en la gestión del desarrollo de la comunidad".

Este proyecto de investigación de la caracterización de las principales problemáticas de las JAC del municipio de Garagoa es un abordaje a la situación actual de estos medios de participación comunitaria de gran relevancia para el desarrollo de la comunidad y transformación de la sociedad.

Dentro de las principales problemáticas que en la actualidad enfrentan estas organizaciones están la debilidad en la comunicación entre los dignatarios, los miembros y la Asamblea general, el desconocimiento de las leyes y estatutos de estas organizaciones, el bajo nivel académico, relevo generacional, limitado nivel de gestión y desconfianza generada por sus dignatarios frente a la comunidad conllevando a que se vuelva apática ante este tipo de escenarios. 
De igual forma, este trabajo de investigación es relevante para el desarrollo social de las organizaciones comunales de base, para futuras indagaciones e investigaciones, dejando como premisa la construcción de un escenario sobre el papel que deben cumplir estas organizaciones en pro de la búsqueda de beneficios sociales, políticos, culturales y sobretodo de recuperación de sentido de pertenencia y prácticas de sus políticas, a su vez se busca que a través de este tipo de investigaciones, promover la participación generando nuevas opciones para los actores sociales involucrados, convirtiéndose en un campo de acción para orientar el que hacer de los líderes comunitarios con fines sociales, comunicativos, educativos y de desarrollo comunitario.

\section{METODOLOGÍA}

Como diseño metodológico para la realización del proyecto se propone la investigación Mixta (cualitativa y cuantitativa), debido a que se orienta en comprender y explorar fenómenos, desde la perspectiva de relacionada con el contexto donde se desarrolla el proyecto y con un enfoque descriptivo, a fin de realizar el diagnóstico y características importantes entorno a las JAC, en este sentido se realizará una encuesta, este instrumento se aplicará a 46 los presidentes de las juntas de acción comunal 29 en el área rural y 17 en el área urbana, como líderes de sus comunidades y de sus JAC, para identificar las percepciones colectivas y caracterizar estrategias; adicional a lo anterior se realizará revisión documental para analizar información relevante, normatividad Colombiana, y análisis de casos exitosos, con el propósito de comprender el contexto de las JAC y las organizaciones comunitarias, para la proyección de estrategias en el fortalecimiento de las mismas.

La investigación se enfoca en dos aspectos primordiales primero en caracterizar las juntas de acción comunal del municipio de Garagoa, para identificar las principales problemáticas y necesidades y en el planteamiento de estrategias que permitan mejorar el liderazgo y gestión, en aspectos como el desarrollo social, cultural y 
económico de las comunidades.

\section{DISCUSIÓN Y RESULTADOS PARCIALES}

La JAC en Colombia está regulada por la ley 743 de 2002 y legitimadas en el artículo 38 de la constitución política, estos organismos de participación comunitaria tienen como principal objetivo implementar modelos de gestión que favorezcan el desarrollo social, cultural y económico de sus comunidades, sin embargo, presentan debilidades que se convierten en barreras y obstáculos para cumplir su finalidad. De acuerdo al (DNP, 2018, pág. 9), en las JAC, existe debilidad en la formación educativa de los dignatarios, la sostenibilidad se ve amenazada por la baja participación, relevo generacional, hay deficiencias en la formulación de proyectos de desarrollo comunitario y la inexistencia de herramientas tecnológicas para manejo de información y articulación con la oferta del estado.

La acción comunal es la organización comunitaria con mayor cobertura geográfica y número de afiliados en Colombia ${ }^{1}$. El municipio de Garagoa cuenta con 46 juntas de acción comunal, ubicadas el $63 \%$ en el área rural y $37 \%$ en el área urbana, con 4200 afiliados, según información del presidente de Asojuntas Garagoa.

De acuerdo a los primeros resultados arrojados por la encuesta a los presidentes de las JAC, se evidencio que de los 46, 29 pertenecen al género masculino y 17 al femenino; la relación en el área urbana el $44 \%$ son hombres y $56 \%$ mujeres y en el área rural el $76 \%$ son hombres y $24 \%$ mujeres, lo cual evidencia que hay mayor participación de las mujeres en el área urbana; la participación de las mujeres refleja

que se ha venido disminuyendo el cierre de brechas de género y liderazgo dentro de sus comunidades.

Figura 1. Edades JAC Garagoa

\footnotetext{
${ }^{1}$ Departamento nacional de planeación (2018) CONPES 3955
} 


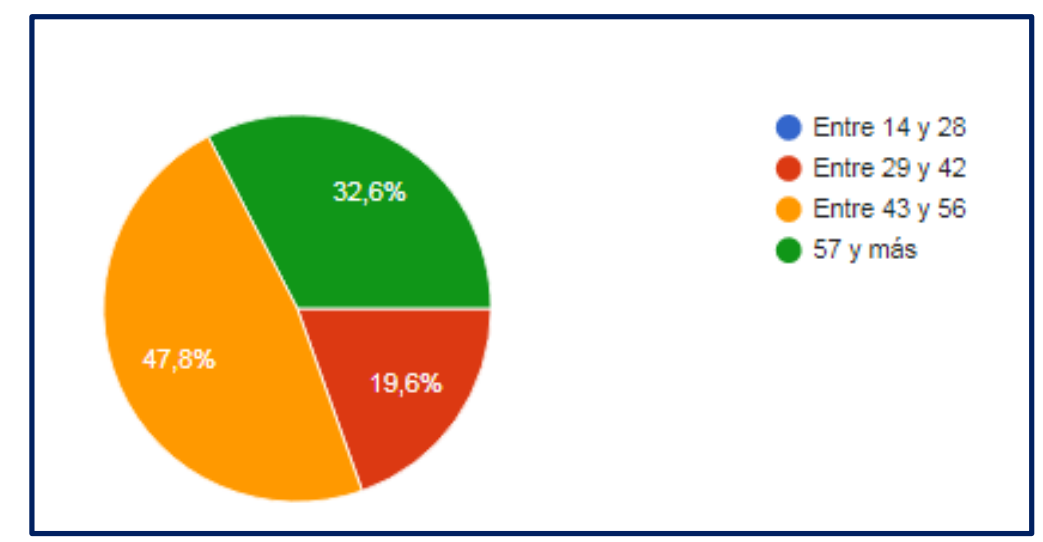

Fuente: elaboración propia

La figura anterior muestra las edades de los presidentes de las JAC Garagoa, donde $19.6 \%$ tienen edades entre los 29 y 42 años, un $47.8 \%$ tienen edades entre los 43 y 56 años y finalmente un $32.6 \%$ son mayores de 57 años, lo cual evidencia que los liderazgos están siendo ejercidos por personas mayores, situación que demuestra la escaza participación de los jóvenes y relevo generacional para la sostenibilidad de las organizaciones comunitarias, por lo cual es importante la promoción e inclusión de nuevos líderes en el escenario comunal.

Figura 2. Antigüedad en la JAC Garagoa

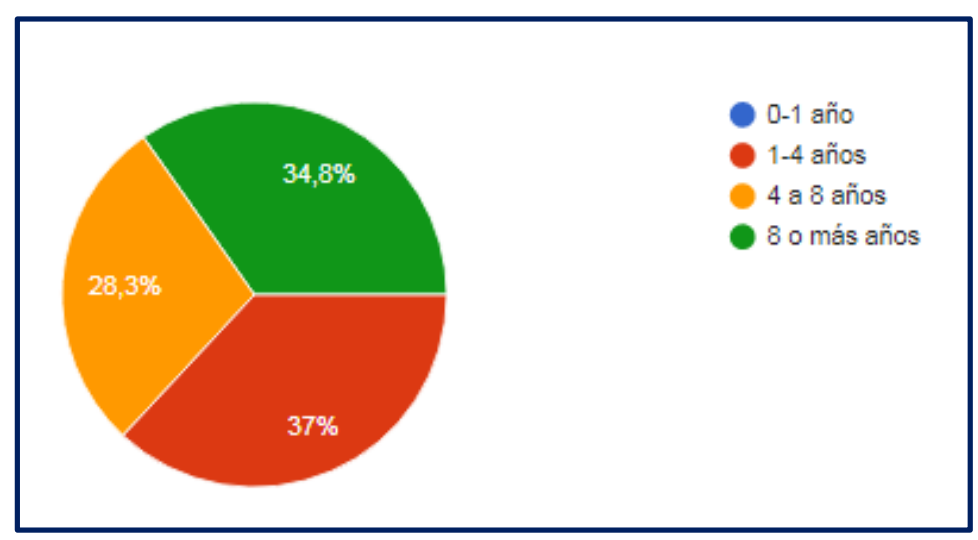

Fuente: elaboración propia 
La figura 2, muestra el tiempo que llevan como miembros de su JAC, donde un $28.3 \%$ están hace 4 a 8 años, el $34.8 \%$ hace más de 8 años y un $37 \%$ entre 1 y 4 años, lo anterior refleja que falta renovación de liderazgo y representatividad, promoción de nuevos líderes empoderados y comprometidos, que promuevan el desarrollo, permanencia y sostenibilidad de las organizaciones.

El ministerio del interior en el año 2017 evidencio que en Colombia aproximadamente el $65 \%$ de los dignatarios figuran hace más de una década, lo que ha generado que la participación en las JAC se concentre en las mismas figuras de poder.

Figura 3. Ocupación dignatarios JAC Garagoa

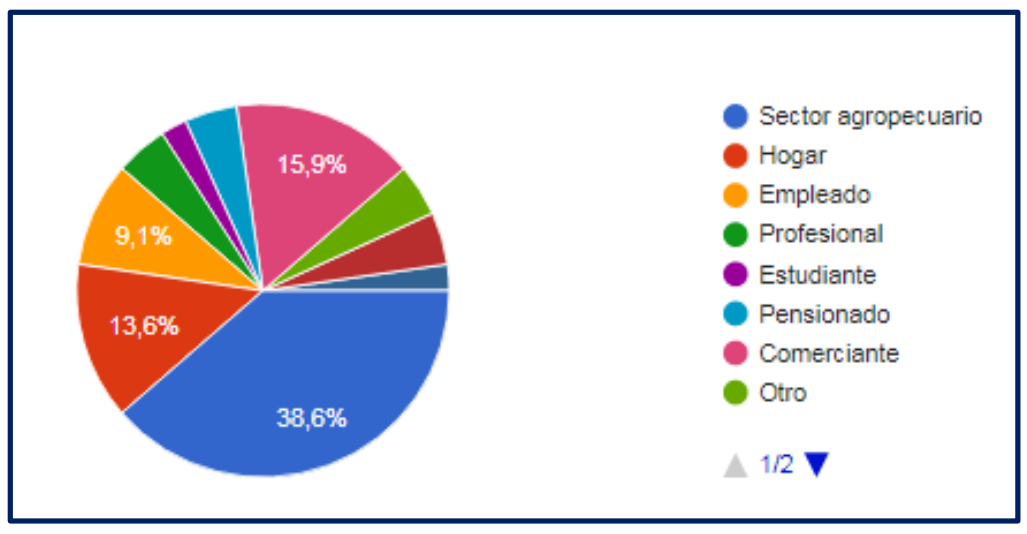

Fuente: elaboración propia

Las ocupaciones de los dignatarios de las JAC Garagoa, 38.6\%, se dedican al sector agropecuario, el $15.9 \%$ son comerciantes, el $13.6 \%$ se dedican a las labores del hogar, el $9.1 \%$ son empleados, el restante $22.6 \%$ se dedican a otras labores como profesional independiente, estudiantes, pensionados e independientes. El resultado anterior muestra tienen una relación directa con la ubicación de las JAC, dado que un $63 \%$ están ubicadas en al área rural por ende sus dignatarios en gran mayoría se dedican a labores del sector agropecuario.

Figura 4. Nivel de educación dignatarios JAC Garagoa 


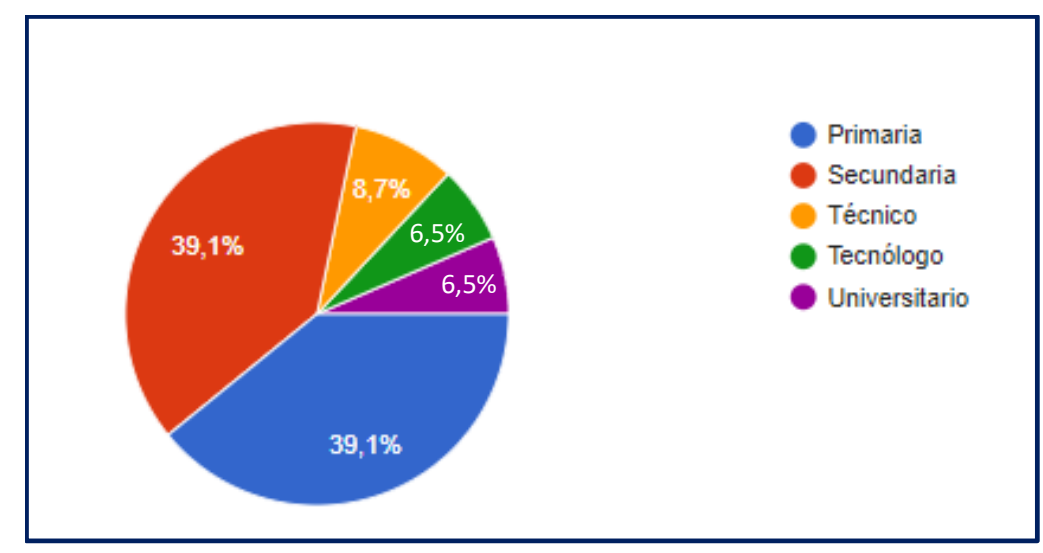

Fuente: elaboración propia

En la figura anterior se muestra el nivel de educación de los dignatarios donde el $39.1 \%$ tiene hasta nivel primaria, el $39.1 \%$ nivel secundario, el $8.7 \%$ son Técnicos, el $6.5 \%$ a tecnólogos y restante $6.5 \%$ son universitarios. Lo anterior refleja un déficit en la educación de los dignatarios, lo cual refleja que su participación y administración en las JAC se debe a su experiencia y conocimientos empíricos y no a su formación educativa, lo que en gran medida afecta el desarrollo de actividades que promueven el desarrollo comunal.

Para el planteamiento de las acciones de generación de estrategias y fortalecimiento de las Juntas de Acción comunal, se continúa con el desarrollo del proyecto de investigación en los aspectos mencionados en el problema de investigación, con el fin de identificar las principales necesidades y debilidades de las organizaciones en aspectos de gestión, administrativos, sociales y financieros.

\section{CONCLUSIONES}

La acción comunal en el municipio de Garagoa enfrenta dificultades que impiden desarrollar ampliamente su objeto social, como lo son el relevo generacional y bajos niveles de formación y competencias, que impactan la gestión administrativa y el 
desarrollo de proyectos, por ende se puede afirmar que en gran medida su participación y administración de las JAC se debe a la experiencia y conocimientos empíricos.

La participación de las mujeres en las JAC, demuestra cómo se ha venido fortaleciendo y cerrado las brechas de género, además de que sus comunidades las reconocen como lideresas, tienen influencia y participan en la toma de decisiones del desarrollo comunitario.

Se evidencia que en las JAC, falta la motivación e inclusión de nuevos actores y líderes, participativos y comprometidos con las dinámicas y desarrollo comunitario, con el propósito de fortalecer la representatividad, sostenibilidad y perdurabilidad de las mismas, por ende debe generarse estrategias para el empoderamiento, relevo generacional y dinamizar actividades que contribuyan al bienestar de la comunidad.

La importancia de caracterizar las JAC, en primer lugar desde aspectos socioeconómicos de los dignatarios, quienes lideran los procesos de la acción comunal y promueven acciones de desarrollo en su comunidad, permite analizar y diagnosticar diversas problemáticas, dentro de las cuales se pueden proponer estrategias para su fortalecimiento participativo, administrativo y de gestión.

\section{REFERENCIAS}

Botero, R. L. (1997). Los sindicatos y las juntas de acción comunal en Colombia. América latina hoy: Revista de ciencias sociales, 17, 87-102. Recuperado de https://dialnet.unirioja.es/servlet/articulo?codigo $=1465709$

CONPES. (2018). Estrategia para el fortalecimiento de la acción comunal en Colombia3955. CONPES, Departamento Nacional de Planeación. Bogotá, Colombia: Consejo 
Nacional de Política Económica y Social - República de Colombia. Recuperado de https://colaboracion.dnp.gov.co/CDT/Conpes/Econ\%C3\%B3micos/3955.pdf

Cano Blandón, L. F. (2008). Gobernabilidad y gobernanza en Medellín: imodelos excluyentes o complementarios de la acción pública en el escenario local? Recuperado de http://hdl.handle.net/10495/2476

Carvajal, Y. L. (2009). Participación ciudadana y buen gobierno: el presupuesto participativo como aporte a la democratización de la ciudad de Medellín. Revista Foro, 68, 105-114. Recuperado de https://search.proquest.com/openview/e9819458b1bba82de9c5be0bc1cd8f2d/1. pdf/

Vélez (2009). Sistemas complejos de gobierno local. Reflexiones sobre la titulación colectiva en el Pacífico vallecaucano. Revista de Estudios Sociales, 32, 74-85. doi: https://doi.org/10.7440/res32.2009.05/

Perea Restrepo, C. M. (2006). COMUNIDAD Y RESISTENCIA poder en lo local urbano. Colombia internacional, 63, 148-171. Recuperado de https://revistas.uniandes.edu.co/doi/abs/10.7440/colombiaint63.2006.07/ 